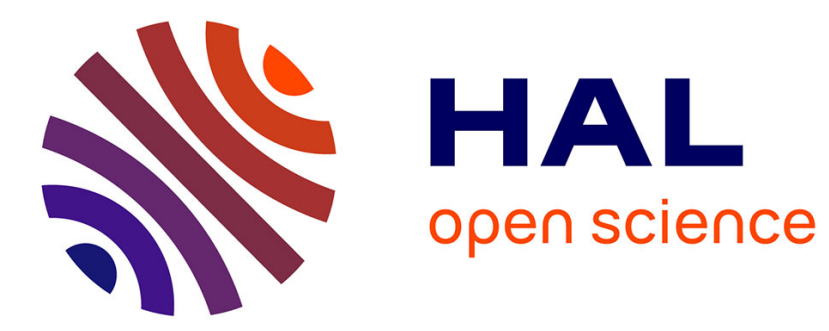

\title{
Methods to determine thermal sensitivity of T-SAGE instrument for MICROSCOPE mission
}

Océane Dhuicque, Manuel Rodrigues, Gilles Métris, Pierre Touboul

\section{To cite this version:}

Océane Dhuicque, Manuel Rodrigues, Gilles Métris, Pierre Touboul. Methods to determine thermal sensitivity of T-SAGE instrument for MICROSCOPE mission. Advances in Space Research, 2021, 68 (4), pp.1989-1997. 10.1016/j.asr.2021.04.019 • hal-03229671

\section{HAL Id: hal-03229671 \\ https://hal.science/hal-03229671}

Submitted on 19 May 2021

HAL is a multi-disciplinary open access archive for the deposit and dissemination of scientific research documents, whether they are published or not. The documents may come from teaching and research institutions in France or abroad, or from public or private research centers.
L'archive ouverte pluridisciplinaire HAL, est destinée au dépôt et à la diffusion de documents scientifiques de niveau recherche, publiés ou non, émanant des établissements d'enseignement et de recherche français ou étrangers, des laboratoires publics ou privés. 
archives-ouvertes

\title{
Methods to determine thermal sensitivity of T-SAGE instrument for MICROSCOPE mission
}

\author{
Océane Dhuicque, Manuel Rodrigues, Gilles Métris, Pierre Touboul
}

\section{To cite this version:}

Océane Dhuicque, Manuel Rodrigues, Gilles Métris, Pierre Touboul. Methods to determine thermal sensitivity of T-SAGE instrument for MICROSCOPE mission. Advances in Space Research, Elsevier, 2021, 10.1016/j.asr.2021.04.019 . hal-03229671

\section{HAL Id: hal-03229671 \\ https://hal.archives-ouvertes.fr/hal-03229671}

Submitted on 19 May 2021

HAL is a multi-disciplinary open access archive for the deposit and dissemination of scientific research documents, whether they are published or not. The documents may come from teaching and research institutions in France or abroad, or from public or private research centers.
L'archive ouverte pluridisciplinaire HAL, est destinée au dépôt et à la diffusion de documents scientifiques de niveau recherche, publiés ou non, émanant des établissements d'enseignement et de recherche français ou étrangers, des laboratoires publics ou privés. 


\title{
Methods to determine thermal sensitivity of T-SAGE instrument for MICROSCOPE mission
}

\author{
Océane Dhuicque ${ }^{\mathrm{a}}$, Manuel Rodrigues $^{\mathrm{a}}$, Gilles Métris ${ }^{\mathrm{b}}$, Pierre Touboul $^{\mathrm{a}}$ \\ a ONERA, chemin de la Hunière, BP 80100, F-91123 Palaiseau Cedex, France \\ ${ }^{b}$ Université Côte d'Azur, Observatoire de la Côte d'Azur, CNRS, IRD, Géoazur, 250 avenue Albert Einstein, F-06560 Valbonne, France
}

\begin{abstract}
MICROSCOPE is a space mission launched in 2016 that aims to test the validity of the Equivalence Principle (EP), the main postulate of General Relativity (GR), with a precision never reached before (Touboul et al. (2001)). EP states that two bodies of different compositions and masses fall with the same acceleration in the same gravitational field. In order to achieve this goal, MICROSCOPE's instrument is composed of two identical electrostatic differential accelerometers. MICROSCOPE will be the first lab to realize an experiment to test the EP in space which allows to break free from perturbations other than gravity. Those tests used to be presented in terms of the Eötvös ratio $\eta$. The first result published in December 2017 shows no evidence of violation higher than $1.3 \times 10^{-14}$ at $1 \sigma$ on $\eta$ (Touboul et al. (2017)). This result was obtained with only $7 \%$ of the whole data, so the statistical uncertainty would be improved with the analysis of the complete data. During the experiment some thermal variations could disturb the estimation of the Eötvös parameter. In order to better estimate the thermal systematic effect, the thermal sensitivity has to be determined. To do so, thermal stimulus are induced on the instrument by means of dedicated heaters and the response in the measured acceleration is analysed. Two methods were applied for theses analysis and will be presented in this paper. The first one operates in the time domain and the second one in the frequency domain. Those methods allow us to obtain a thermal systematic of $8.6 \times 10^{-16}$ which represents an improvement by one order of magnitude with respect to the result of Touboul et al. (2017).
\end{abstract}

Keywords: thermal sensitivity ; signal processing ; data analysis ; MICROSCOPE ; space accelerometer

\section{Introduction : The Equivalence Principle}

The Equivalence Principle (EP) states the equivalence of the inertial and the gravitational masses. Referring to the newtonian formulation of dynamics and gravitation, the inertial mass quantifies the resistance of a body to a modification of its motion as answer to all kind of forces and the gravitational mass quantifies the gravitational force between bodies. A priori there is no reason for theses masses to be identical: for example their ratio could depend on the atomic composition of the bodies or on the quantity of matter they contain. However, in 1610, Galileo demonstrated implicitly (the concept of inertial and gravitational mass appeared a few decades later with Newton)

Email address: oceane.dhuicque@onera.fr (Océane Dhuicque) this equality of masses with his rudimentary experiment which consisted to bowl down weights on inclined boards and to compare their free fall. Hence the EP relies on experimental facts and is not an untouchable concept of physics. However, it is a pillar of General Relativity (GR) which predicted a lot of new physical effects which have been verified by experiments. The EP was tested by means of several experiments this last century searching for a significant deviation of the Eötvös parameter from 0 . The Eötvös parameter is defined, for two bodies $A$ and $B$, by the ratio:

$$
\eta(A, B)=\frac{\frac{m_{G, A}}{m_{I, A}}-\frac{m_{G, B}}{m_{I, B}}}{\frac{1}{2}\left(\frac{m_{G, A}}{m_{I, A}}+\frac{m_{G, B}}{m_{I, B}}\right)}
$$

where the subscripts $I$ and $G$ indicate the inertial and the gravitational masses respectively. In particular, using the torsion- 
4 balance test of the university of Washington, Wagner et al. 5 (2012) found no violation for several pairs of materials, with 6 an accuracy of about $10^{-13}$ on $\eta$. Using the lunar laser ranging 7 (Viswanathan et al. (2018)) has even yielded a slightly better ac8 curacy but with a different interpretation because this tests both 9 the impact of the difference of composition between Earth and 10 Moon and the impact of their self energy, the so called Nordvedt 11 effect (Nordtvedt (1968)).

12 Despite all the success of GR, several alternative theories 13 are under studies, aiming to unify gravitation with quantum 14 physics. Contrary to GR, most of these theories do not im15 pose the EP. That is why, testing the EP with the best accuracy 16 possible is an interesting way to question theses theories. This 17 was the main goal of the MICROSCOPE space mission which 18 operated between April 2016 and October 2018.

19 The paper is organized as follows. In section 2, we give 21 an overview of the MICROSCOPE experiment. In section 3 we 22 explain the need for a thermal characterization of the instrument 23 and the principle to achieve this. The analysis methods used to 24 estimate the thermal sensitivity will be presented in section 4 . 25 Section 5 will show the results obtained with these methods.

\section{6}

\section{2. MICROSCOPE: a lab in space}

\section{2.1. The MICROSCOPE space mission}

31 MICROSCOPE is the first experiment to test the equiva32 lence principle in space, away from disturbances such as seis33 mic noise or laboratory Earth gravity field fluctuations. Several 34 space projects with the same aim have been studied in the past 35 but not realized. We can quote the work of Chapman \& Hanson 36 (1970) in the 1970s that we better know as the STEP experi37 ment from Stanford University (Mester et al. (2001)) or later 38 the work of Everitt et al. (2003) or Nobili et al. (1995) from 39 Pisa University. MICROSCOPE was launched in April 2016 40 in a sun-synchronous orbit and was decommissioned in Octo41 ber 2018. In space, the experiment is not limited by the on42 Earth perturbations anymore and in orbit free fall is very pure 43 and almost infinite thanks to the drag-free system (Robert et al. $45(2020))$.

46

47 2.2. Mission payload: T-SAGE

48 The instrument at the heart of the experiment is composed 49 of two identical electrostatic differential accelerometers. The

50 first one, SUREF standing for Sensor Unit REFerence, consists 51 of one inner mass and one outer mass made of PtRh, a $90 \%$ 52 platinum-10\% rhodium alloy. The SUEP, standing for Sensor 53 Unit for the Equivalence Principle, has its inner mass made 54 of the same material as SUREF inner mass and its outer mass 55 made of TA6V, a $90 \%$ titanium- $6 \%$ aluminum- $4 \%$ vanadium al56 loy (Figure 1). Both masses are kept in equilibrium thanks to a 58 control loop and to several electrodes that allow first to detect 59 their motions and second to apply the appropriate electrostatic 60 forces to keep them at rest with respect to the satellite. These 61 electrostatic forces compensate all other external forces and 62 the applied voltage allows to calculate the acceleration which 63 would have been experienced by the masses in absence of the electrostatic forces. This is what we will call simply (and abusively since the masses are at rest) the measured acceleration.

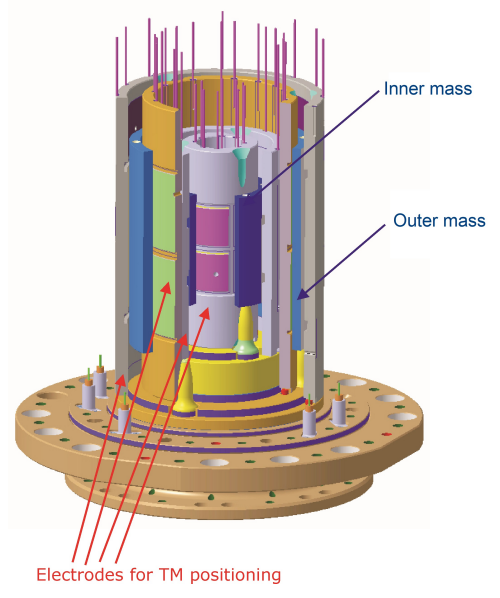

Fig. 1. Configuration of one sensor unit (SU)

\subsection{The measurement model}

Since we want to compare the free fall of two test masses, we are interested in the difference of acceleration measured between these two masses, $\vec{\Gamma}_{d}^{\text {meas }} \equiv \vec{\Gamma}_{1}^{\text {meas }}-\vec{\Gamma}_{2}^{\text {meas }}$, 1 refers to the inner mass and 2 to the outer mass of one Sensor Unit. A more detailed mathematical model of this difference is developed in Touboul et al. (2020) but here we use a simplified expression which is more than enough for our needs:

$$
\vec{\Gamma}_{d}^{\text {meas }}=\left[M_{c}\right]\left(\delta(2,1) \vec{g}\left(O_{\text {sat }}\right)+\left([T]-\left[I_{n}\right]\right) \vec{\Delta}\right)+2\left[M_{d}\right] \vec{\Gamma}_{c}^{a p p}+\vec{B}_{0}+\vec{\Gamma}_{d}^{(n)}+\Gamma_{d}^{(s)}
$$

This equation can be understood in the following way:

- $\vec{g}\left(O_{\text {sat }}\right)$ is the gravity acceleration at the center of mass of the satellite and $\delta(2,1)$ is defined as

$$
\delta(2,1)=\frac{m_{G, 2}}{m_{I, 2}}-\frac{m_{G, 1}}{m_{I, 1}}
$$

$\delta(2,1)$ is close to the Eötvös parameter $\eta(2,1)$ defined in Eq. 1 since $\frac{m_{G}}{m_{I}}$ does not differ from 1 by a quantity larger than $10^{-13}$ as demonstrated by previous experiments quoted in Section 1 ; thus $\delta(2,1) \vec{g}\left(O_{\text {sat }}\right)$ quantifies the possible EP violation signal.

- The centers of the 2 test masses are separated by the vector $\vec{\Delta}$ which leads to the gravity gradient $[T] \vec{\Delta}$ and to the acceleration of inertia $-\left[I_{n}\right] \vec{\Delta}$. Here $[T]$ is the gravity gradient tensor and $\left[I_{n}\right]=[\Omega]^{2}+[\dot{\Omega}]$ where $[\Omega]$ is the angular velocity tensor that can be defined by the operator $\vec{\Omega} \times$.

- These two terms constitute the theoretical "applied" differential acceleration $\vec{\Gamma}_{d}^{a p p}$ which would be measured by a perfect instrument; $\left[M_{c}\right]$ and $\left[M_{d}\right]$ are matrices which gather the linear transformation between the "applied" acceleration and the measured acceleration: rotations, scale factors and couplings between axis; $\left[M_{c}\right]$ (close to the identity matrix) is the component common to the two masses and multiplies the "applied" differential acceleration. 
- $\vec{\Gamma}_{c}^{a p p}$ is the applied acceleration common to the two test masses; in case of perfectly identical instruments it would have no contribution to the measured differential acceleration but in practice it has a small projection $\left[M_{d}\right] \vec{\Gamma}_{c}^{a p p}$ due to the relative difference of characteristics between the two test masses.

- $\overrightarrow{B_{0}}$ is the DC acceleration bias due to electronics offsets and to parasitic forces.

- $\vec{\Gamma}_{d}^{(n)}$ is the acceleration measurement noise.

- $\vec{\Gamma}_{d}^{(s)}$ represents the systematic thermal effects on which we will focus in this paper.

The axis collinear to the axis of symmetry of the cylindrical test masses, labelled $x$, is about one order of magnitude more accurate than the radial axis. That is why the analysis is restricted to the $x$ axis and the projection of Eq. 2 on this axis has been considered. We introduce additional simplifications not used in the real analysis but allowing a better understanding of the main effects: we keep only the first components of the matrix $\left[M_{c}\right]$ and $\left[M_{d}\right]$ which are renamed $K_{c, x}$ and $K_{d, x}$ respectively; moreover, the theoretical applied common acceleration $\Gamma_{c, x}^{a p p}$ is approximated by its measurement $\Gamma_{c, x}^{\text {meas }}$. At this point we have to precise that the measured acceleration $\vec{\Gamma}_{c}^{\text {meas }}$ can be controlled and minimized thanks to the drag-free system through cold gas thrusters (Robert et al. (2020)). We are left with the model :

$$
\begin{aligned}
\Gamma_{d, x}^{\text {meas }}= & K_{c, x}\left[\delta(2,1) g_{x}\left(O_{s a t}\right)+S_{x x} \Delta_{x}+S_{x y} \Delta_{y}+S_{x z} \Delta_{z}\right] \\
& +2 K_{d, x} \Gamma_{c, x}^{\text {meas }}+B_{0, x}+\Gamma_{d, x}^{(n)}+\Gamma_{d}^{(s)}
\end{aligned}
$$

where $[S]=[T]-\left[I_{n}\right]$. The payload is embedded in the satellite which can have different pointing configurations. During all but a few scientific sessions, the $x$ axis is maintained parallel to the mean orbital plane. For most of the calibration sessions the pointing was almost inertial in the sense that it just follows the slow precession of the orbital plane. During the sessions dedicated to the EP test, the satellite was spinning about the $y$ axis normal to the orbital plane with a frequency $f_{\text {spin }}$. Consequently, $g_{x}$ (the projection of the gravity acceleration on the $x$ axis) varies at the frequency $f_{E P}=f_{\text {spin }}+f_{\text {orb }}$. After the commissioning phase of the MICROSCOPE mission, it was decided to select, among the preprogrammed values for $f_{E P}$, $f_{E P 2}=9.24 \times 10^{-4}$ and $f_{E P 3}=3.11 \times 10^{-3}$.

The goal of the MICROSCOPE experiment is to use the measured acceleration in order to estimate the parameter $\delta(2,1)$. To do this it is necessary to quantify the other terms of Eq. 4 This has been done by means of various dedicated sessions called calibration sessions with a specific configuration for each parameter. Furthermore, other more subtle perturbations could also affect the measurement. During the analysis of the first measurements (Touboul et al. (2017), Touboul et al. (2019)), it appeared that the thermal sensitivity of the instrument, based on a very conservative evaluation, was the main source of systematic errors. At that time, since the measurement sessions dedicated to the more precise estimation of the thermal sensitivities had still not been finished, preliminary analyses were taken into account.

\section{Characterizing the thermal sensitivity of the instrument}

\subsection{Thermal configuration of the instrument}

The interface control unit included the digital controller, the Front End Electronic Unit (FEEU) that contained the reference voltages and the measurement pick up and the Sensor Unit (SU) that houses the test masses. All these elements have their own temperature during nominal operation and variations of each of these temperatures might impact the measurement. In order to characterize the instrument's thermal behavior we have access to the temperatures of the different parts of the instrument through various probes located inside or on the skin of each element.

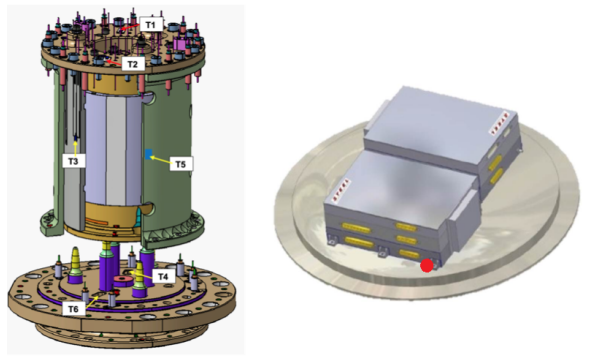

Fig. 2. Left : SU configuration : T1, T2; T3, T4, T5 and T6 are the temperature probes locations ; Right : FEEU configuration : red spot represents the interface temperature sensor

For the FEEU, the temperature fluctuations have been specified to $0.5 \mathrm{~K} / \sqrt{\mathrm{Hz}}$ and its systematic variations specified to $3 \mathrm{mK}$ at $f_{E P}$. One Pt-resistance (Pt1000) is on the circuit board and at the unit interface (Figure 2) with a probe noise of $20 \mathrm{mK} / \sqrt{\mathrm{Hz}}$. Figure 3 shows the square root of the measured temperature PSD during a scientific session. We can see (and this is confirmed by a numerical estimation) that there is no signal emerging from the noise around the frequency $f_{E P}$. This is still the case even when we cumulate several sessions with a total duration of 320 orbits which last 5946s each. This means that a signal which could be hidden in this noise could not have an amplitude larger than $0.02 / \sqrt{320 \times 5946} \simeq 14 \times 10^{-6} \mathrm{~K}$. To be conservative we retain the upper bound of $20 \mu \mathrm{K}$.

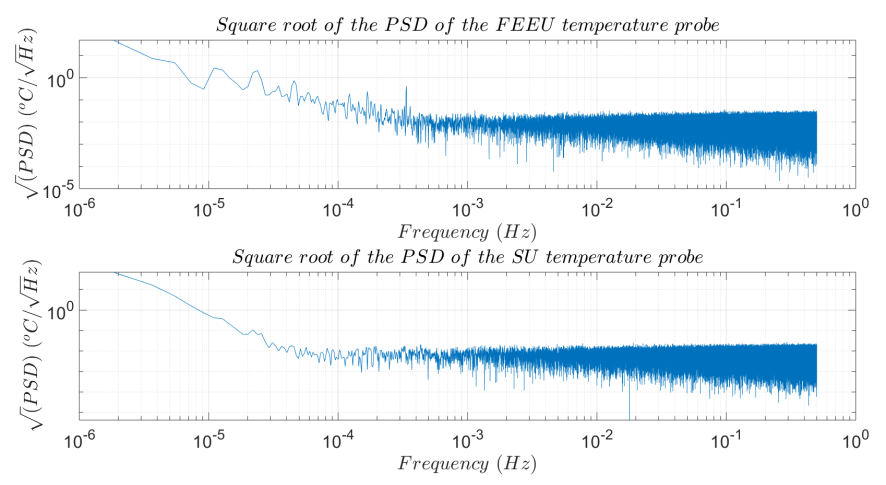

Fig. 3. Square root of the Power Spectrum Density Spectral of the Pt1000 probe during science sessions for the FEEU (top) and for the SU (bottom)

For the SU part, the specifications for the thermal stability are $0.3 \mathrm{~K} / \sqrt{\mathrm{Hz}}$ and its systematic to $1 \mathrm{mK}$ at $f_{E P} .6 \mathrm{Pt} 1000$ 
3

4 probes, with a probe noise of $20 \mathrm{mK} / \sqrt{\mathrm{Hz}}$, are placed at 6 dif5 ferent locations on the SU part (Figure 2). The analysis of sev6 eral sessions up to 320 orbits duration (Touboul et al. (2017)), 7 establishes that there is no temperature signal emerging from 8 the noise at $1 \sigma$. The SU temperature is the mean of two 9 probes'measures, this allows us to put an upper bound on the 10 thermal systematic variation at $f_{E P}$ to $15 \mu \mathrm{K}$ as we can divide 11 the upper bound specified for the FEEU by a factor $\sqrt{(2)}$.

\section{2}

13

14

\section{3.2. Equation for the thermal model}

16

17

18 As indicated above, the two main components for which 19 variations of temperature are likely to induce variations of ac20 celerometric measurements are the mechanical part (the SU) 21 and its FEEU. That is why our model will include the sensitivi22 ties to these temperatures:

27 where $\Delta T_{S U}$ and $\triangle T_{F E E U}$ mean that we consider only the vary28 ing parts of the temperatures; in practice we get these variations 29 by subtracting the mean value of the temperature $\bar{T}$ to the mea30

35 In practice $T_{S U}$ will be computed as the mean of the probes $36 T_{1}$ and $T_{4}, T_{S U}=\frac{\left(T_{1}+T_{4}\right)}{2}$, representing the best guess of the SU 37 interface temperature.

38 The most natural way to estimate $\lambda_{S}$ and $\lambda_{F}$ would be to do 39 it directly during the scientific sessions by looking for a cor$4 \odot$ relation between the measured acceleration and the measured 41 temperature. But in our case the temperature is so stable during 42 these sessions that we observe no variations around the relevant 43 frequencies in the limit of the noise of the probes. Thus this 45 classical method would lead to a null effect. Our approach is 46 more conservative: we assume that a systematic fluctuation of 47 the temperature could be hidden in the measurement noise of 48 the probes and compute the upper bound of the thermal effects 49 by multiplying this maximum amplitude that we could not see 50 by the thermal sensitivity; but to observe this thermal sensitiv51 ity it is necessary to create artificially very large variations of 52 temperature. This is what is done during the thermal sessions.

55

\section{3.3. Thermal sessions}

59 Thermal sessions run during the mission consist in introduc$6 \odot$ ing a temperature stimulus at a frequency $f_{s t i}$ (close to $f_{E P}$ ) near 61 the sensor unit or near the electronic unit. This stimulus is in62 duced by different double layers heaters placed at different lo63 cation in the SU and in the FEEU (Figure 4).

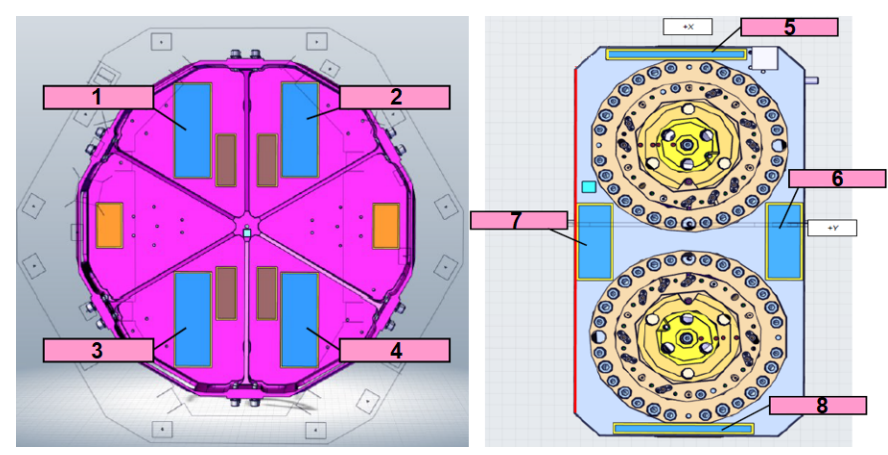

Fig. 4. Heaters 1 to 4 are on the interface of the FEEU (left) and heaters 5 to 8 are near the $\mathrm{SU}$ (right) in blue

These sessions are performed in inertial mode, which corresponds to the worst case for thermal fluctuations because when the satellite is in spinning mode, the temperature stability is better due to the thermal filtering more efficient at higher frequency.

\begin{tabular}{lllll} 
Session & Part of the instrument tested & $T_{0}$ & $T_{s t i}(s)$ & $T_{i}(s)$ \\
\hline $\mathbf{2 6 6}$ & SUEP FEEU & $8 \mathrm{~h} 00 \mathrm{~m} 00 \mathrm{~s}$ & 1500 & 300 \\
$\mathbf{2 7 0}$ & SUEP SU & $8 \mathrm{~h} 00 \mathrm{~m} 00 \mathrm{~s}$ & 4500 & 500 \\
$\mathbf{2 9 8}$ & SUREF FEEU & $4 \mathrm{~h} 00 \mathrm{~m} 45 \mathrm{~s}$ & 321 & 64 \\
$\mathbf{3 0 0}$ & SUREF SU & $4 \mathrm{~h} 00 \mathrm{~m} 45 \mathrm{~s}$ & 321 & 64 \\
$\mathbf{3 0 4}$ & SUREF FEEU & $4 \mathrm{~h} 12 \mathrm{~m} 28 \mathrm{~s}$ & 1082 & 200 \\
$\mathbf{3 0 6}$ & SUREF SU & $4 \mathrm{~h} 12 \mathrm{~m} 28 \mathrm{~s}$ & 1082 & 120 \\
$\mathbf{3 1 4}$ & SUEP FEEU & $4 \mathrm{~h} 12 \mathrm{~m} 28 \mathrm{~s}$ & 1082 & 200 \\
$\mathbf{3 1 6}$ & SUEP SU & $4 \mathrm{~h} 12 \mathrm{~m} 28 \mathrm{~s}$ & 1082 & 120 \\
$\mathbf{3 2 0}$ & SUEP FEEU & $4 \mathrm{~h} 00 \mathrm{~m} 45 \mathrm{~s}$ & 321 & 64 \\
$\mathbf{3 2 2}$ & SUEP SU & $4 \mathrm{~h} 00 \mathrm{~m} 45 \mathrm{~s}$ & 321 & 64 \\
$\mathbf{7 5 8}$ & SUEP FEEU & $8 \mathrm{~h} 00 \mathrm{~m} 00 \mathrm{~s}$ & 321 & 128 \\
$\mathbf{7 6 0}$ & SUEP FEEU & $8 \mathrm{~h} 00 \mathrm{~m} 00 \mathrm{~s}$ & 1082 & 432 \\
$\mathbf{7 8 2}$ & SUREF SU & $8 \mathrm{~h} 00 \mathrm{~m} 00 \mathrm{~s}$ & 4500 & 500
\end{tabular}

Table 1. List of thermal sessions run during the experiment and their characteristics. $T_{0}$ is the duration of the session, $T_{s t i}$ is the period of the thermal heating cycle and $T_{i}$ is the duration of the heating phase inside each periodic stimuli.

Two kinds of session were run dedicated either to the thermal characterization of the SU or of the FEEU. In each session the heaters of the SU, respectively the FEEU, are switched on during a series of time intervals of duration $T_{i}$. The beginnings of these intervals are separated by a duration $T_{s t i}$. The resulting thermal profile is shown in Figure 5
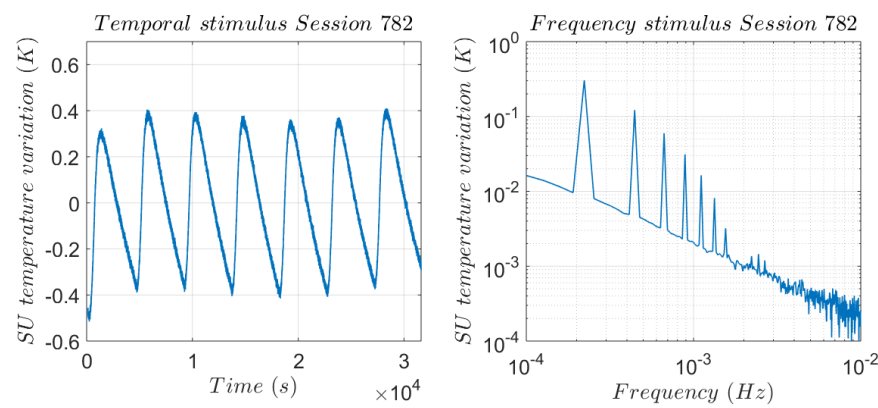

Fig. 5. Temperature stimulus as a function of time (left) and its discrete Fourier transform (right) for session 782

Several constraints have imposed the choice of the heating 
frequency $f_{s t i}=\frac{1}{T_{s t i}}$. First of all $f_{s t i}$ is defined as a non-multiple of $f_{\text {orb }}$ in order to avoid the combination of $f_{\text {orb }}$ and $f_{\text {sti }}$ while testing the thermal sensitivity of the instrument. If $f_{s t i}$ is too high, $T_{s t i}$ is short and thermal variations too small to be seen. A long period $T_{s t i}$ improves the signal to noise ratio, but if the heating time $T_{i}$ is too long it would be difficult to dissipate the heat in the satellite. And last but not least, we need to add a minimum number of cycles, defined by $\frac{T_{0}}{T_{s t i}}$.

The list of the thermal sessions is presented in Table 1 .

\section{Analysis methods}

\subsection{Correction and pre-processing of the differential acceler- ation}

By inserting Eq. 5 in Eq. 4 one have the following measurement equation :

$$
\begin{aligned}
\Gamma_{d, x}^{\text {meas }}= & K_{c, x}\left[\delta(2,1) g_{x}\left(O_{s a t}\right)+S_{x x} \Delta_{x}+S_{x y} \Delta_{y}+S_{x z} \Delta_{z}\right] \\
& +2 K_{d, x} \Gamma_{c, x}^{\text {meas }}+B_{0, x}+\Gamma_{d, x}^{(n)} \\
& +\lambda_{S} \Delta T_{S U}+\lambda_{F} \Delta T_{F E E U}
\end{aligned}
$$

During the EP sessions, the experiment has been designed so that the thermal effect (last line of Eq. 7) remains as small as possible (in particular the temperature is very stable). On the contrary during thermal sessions, this contribution must be dominant, around the frequency $f_{s t i}$ and its harmonics, in order to characterize the sensitivities. Indeed all other terms of Eq. 7 are much smaller. The term $2 K_{d, x} \Gamma_{c, x}^{\text {meas }}$ gives a contribution at the orbital frequency quite important although not as $f_{s t i}$. This is due to the fact that during thermal sessions the drag-free system is not activated and $\Gamma_{c, x}^{\text {meas }}$ includes in particular the satellite drag by the atmosphere. Since we are in inertial pointing the drag will be visible in the signal at the orbital frequency which is not very far from $f_{s t i}$ for some thermal sessions. That is why the measured differential acceleration must be corrected from this effect before searching for the imprint of the thermal variations:

$$
\Gamma_{d, x}^{c o r r}=\Gamma_{d, x}^{\text {meas }}-2 \hat{K}_{d, x} \Gamma_{c, x}^{\text {meas }}
$$

The correction is very simple since $\Gamma_{c, x}^{\text {meas }}$ is obtained directly from the measured accelerations and the scale factors $K_{d, x}$ have been estimated during dedicated calibration sessions: $K_{d}($ SUREF $)=-1.45 \times 10^{-2}$ and $K_{d}(S U E P)=8.5 \times 10^{-3}$.
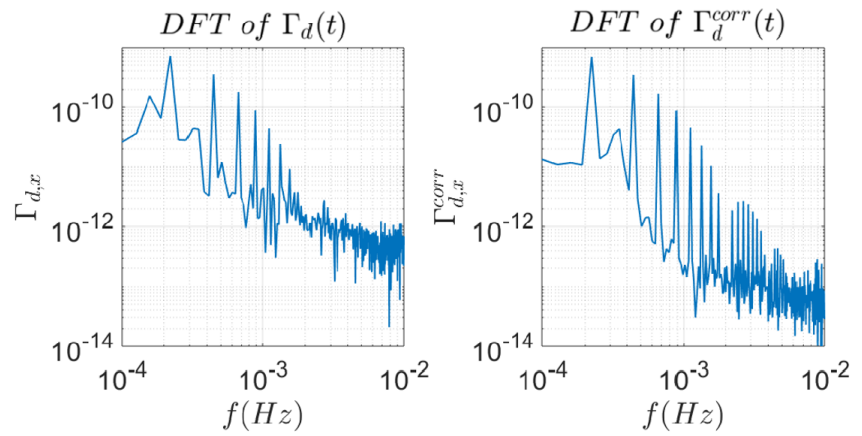

Fig. 6. Discrete Fourier transform of the differential acceleration for session 782 before (left) and after (right) correction of the common mode projection expressed in Eq. 8
Figure 6 shows the DFT of $\Gamma_{d, x}^{\text {meas }}$ before and after this correction.

We complete the preparation of the data with the following pre-processing:

- Some measured acceleration data have been identified on board as corrupted due to missing data. They are very rare (typically 1 over 100000 data samples) and, to keep a perfect regularity in the sampling, we have chosen to reconstruct these data as the mean of the 10 neighboring points. We have checked that any other reasonable reconstruction does not change our final results.

- To get rid of the long term evolution in the measured accelerations and temperatures, polynomials of degree 2 are fitted and subtracted to these signals.

- For each thermal session we will extract the longest segment lasting exactly a whole number of periods $T_{s t i}$; the processing will be performed on this segment.

After these correction and pre-processing, we can consider that the residual measured differential acceleration is clean enough to be represented by means of the thermal variations:

$$
\Gamma_{d, x}^{c o r r} \approx \lambda_{S} \Delta T_{S U}+\lambda_{F} \Delta T_{F E E U}
$$

\subsection{Temporal method}

Observing the DFT of the corrected acceleration Figure 7. we see that it features a pattern similar to the temperature DFT : we have a series of peaks at frequencies multiple of $f_{\text {sti }}$.
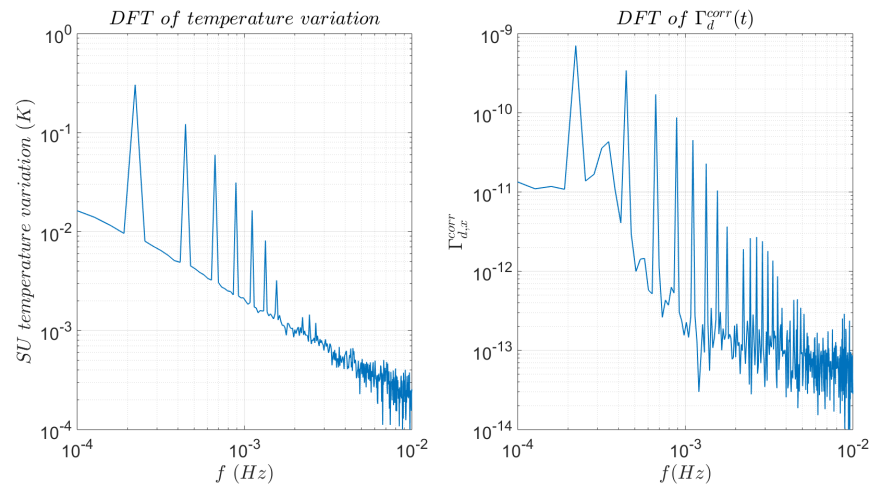

Fig. 7. DFT of temperature variation (left) and DFT of corrected differential acceleration (right)

To estimate the sensitivity, the simplest idea is to compare the amplitudes of the peaks of the acceleration on one hand and of the temperature on the other hand. More precisely, for each of these data, we fit the model :

$$
\text { Model }=\sum_{k=1}^{n}\left[A_{k} \times \sin \left(2 k \pi f_{s t i} t\right)+B_{k} \times \cos \left(2 k \pi f_{s t i} t\right)\right]+C
$$

The number of frequencies multiple of $f_{s t i}$ taken into account, $n$ in Eq. 10, depends on the duration of the session to reject the stochastic noise and on the amplitude of the stimuli. The temperature and the acceleration measurement are filtered using the 
3

4 same low-pass filter with a cutoff frequency fixed to $9 f_{\text {sti }}$ in or5 der to get rid of the high frequency noise and to preserve the 6 low frequency part of interest for our goal. Then the coeffi7 cients $A_{k}, B_{k}$ and $C$ are estimated by means of a least-square 8 regression. Since the measurements of temperature as well as 9 acceleration are affected by a non white noise, the least squares 10 solution has the minimum variance only if one uses the appro11 priate non-diagonal weighting. Even though we use this kind 12 of solutions or equivalent ones (Baghi et al. (2015), Baghi et al. 13 (2016), Bergé et al. (2020)) in the analysis of scientific ses14 sions, this heaviness is not justified here: the important point 15 is that our estimator is unbiased, which is the case of the ordi16 nary least squares estimator. Figure 8 shows examples of such 17 fittings.

18

53 and then

$$
\sigma_{M_{k}}=\sqrt{\left(\frac{A_{k}}{M_{k}}\right)^{2} \sigma_{A_{k}}^{2}+\left(\frac{B_{k}}{M_{k}}\right)^{2} \sigma_{B_{k}}^{2}}
$$

to the temperature variation $\Delta T$. From there Eq. 9 in the time domain is transformed into an equation in the frequency domain:

$$
\Gamma_{d, x}^{c o r r}(f)=\lambda_{S} \Delta T_{S U}(f)+\lambda_{F} \Delta T_{F E E U}(f)
$$

where $\Gamma_{d, x}^{c o r r}, \Delta T_{S U}$ and $\Delta T_{F E E U}$ are given at the discrete frequencies $f_{q}=\frac{q}{T}=\frac{q}{n T_{s i}}=\frac{q}{n} f_{s t i}$. In particular, for $q=k n$, we get the harmonics of $f_{s t i}: f_{q}=f_{k n}=k f_{s t i}$. Since the stimuli of the temperature is at the frequencies $=k f_{\text {sti }}$, we will focus on observations around these frequencies: we use a system of equations as in Eq. 15 applied to these frequencies. Then this system is inverted by means of a least-square method simultaneously on each neighboring frequency of interest $=k f_{s t i}$.

This strategy has several advantages:

1. the estimation is no longer strictly limited to the frequency $f_{s t i}$ and its harmonics; if, for a given reason, there is a thermal signal close to these frequencies, we can capture it;

2. on the contrary, using only frequencies in the neighboring of $f_{s t i}$ or its harmonics ensures that our model will not be disrupted by other effects (because $f_{\text {sti }}$ has been chosen outside of other natural frequencies).

The number of neighboring frequencies is chosen so that the bands around 2 successive harmonics do not overlap. Basically we consider 3 points before and after the frequency of interest. The drawback is that with this limited number of equations the computation of the variance is not reliable. The Figure 9 shows the amplitudes of the DFT for the temperature on the left panel and for the acceleration on the right panel. The different colors locate the frequency bands associated to each harmonic.
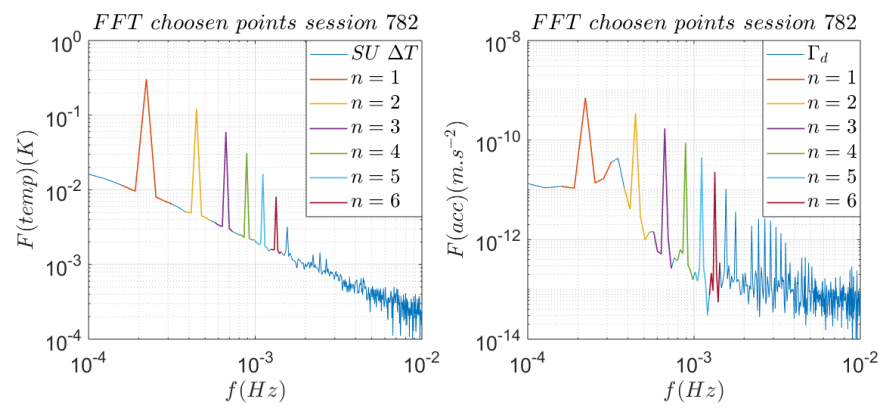

Fig. 9. Discrete Fourier transform of the temperature variation (left) and of the differential acceleration (right) for session 782

\section{Results and discussion}

The data are processed with the methods presented below. For each stimuli's frequency a thermal sensitivity is estimated since a frequency dependency is assumed, in particular for the instrument SUEP due to the fact that its fixing system in the satellite is different from the SUREF one. We can also note that the methods used will give us a positive value as we consider amplitudes of the signal for the temporal method and the norm of the DFT for the frequency method. In fact, we did not want to correct the measurement from the thermal effect but only estimate a maximum value of its impact. 


\begin{tabular}{llllll} 
Session & $k$ & $k f_{s t i}$ & $\lambda_{F f}$ & $\lambda_{F t}$ & $\sigma_{t}$ \\
\hline 266 & 1 & $6.67 \times 10^{-4}$ & $8.21 \times 10^{-11}$ & $7.90 \times 10^{-11}$ & $1.3 \times 10^{-12}$ \\
$\mathbf{7 6 0}$ & 1 & $9.24 \times 10^{-4}$ & $7.29 \times 10^{-11}$ & $6.32 \times 10^{-11}$ & $4.6 \times 10^{-12}$ \\
$\mathbf{2 6 6}$ & 2 & $1.33 \times 10^{-3}$ & $4.46 \times 10^{-11}$ & $5.12 \times 10^{-11}$ & $2.2 \times 10^{-12}$ \\
$\mathbf{7 5 8}$ & 1 & $3.11 \times 10^{-3}$ & $5.52 \times 10^{-11}$ & $4.79 \times 10^{-11}$ & $7.4 \times 10^{-12}$
\end{tabular}

Table 2. Sensitivity of the FEEU of the SUEP instrument with both methods for the frequency $k f_{s t i} ; \lambda_{F f}:$ sensitivity computed with the frequency method; $\lambda_{F t}$ : sensitivity computed with the temporal method; $\sigma_{t}$ the standard deviation computed using the temporal method as in Eq. 14

\begin{tabular}{llllll} 
Session & $k$ & $k f_{s t i}$ & $\lambda_{F f}$ & $\lambda_{F t}$ & $\sigma_{t}$ \\
\hline 304 & 1 & $9.24 \times 10^{-4}$ & $2.18 \times 10^{-11}$ & $2.59 \times 10^{-11}$ & $1.7 \times 10^{-12}$ \\
304 & 2 & $1.85 \times 10^{-3}$ & $7.12 \times 10^{-11}$ & $4.02 \times 10^{-11}$ & $4.3 \times 10^{-12}$ \\
304 & 3 & $2.78 \times 10^{-3}$ & $4.02 \times 10^{-11}$ & $9.31 \times 10^{-12}$ & $9.9 \times 10^{-12}$ \\
$\mathbf{2 9 8}$ & 1 & $3.11 \times 10^{-3}$ & $1.58 \times 10^{-10}$ & $1.5 \times 10^{-10}$ & $7.5 \times 10^{-11}$
\end{tabular}

Table 3. Same as Table 2 for the FEEU of the SUREF instrument

\begin{tabular}{llllll} 
Session & $k$ & $k f_{\text {sti }}$ & $\lambda_{F f}$ & $\lambda_{F t}$ & $\sigma_{t}$ \\
\hline $\mathbf{2 7 0}$ & 1 & $2.22 \times 10^{-4}$ & $2.14 \times 10^{-8}$ & $2.15 \times 10^{-8}$ & $8.8 \times 10^{-12}$ \\
$\mathbf{2 7 0}$ & 2 & $4.44 \times 10^{-4}$ & $1.67 \times 10^{-8}$ & $1.67 \times 10^{-8}$ & $1.9 \times 10^{-11}$ \\
$\mathbf{2 7 0}$ & 3 & $6.66 \times 10^{-4}$ & $1.42 \times 10^{-8}$ & $1.39 \times 10^{-8}$ & $3.6 \times 10^{-11}$ \\
$\mathbf{2 7 0}$ & 4 & $8.88 \times 10^{-4}$ & $1.24 \times 10^{-8}$ & $1.15 \times 10^{-8}$ & $6.5 \times 10^{-11}$ \\
$\mathbf{3 1 6}$ & 1 & $9.24 \times 10^{-4}$ & $1.4 \times 10^{-8}$ & $1.4 \times 10^{-8}$ & $5.0 \times 10^{-11}$ \\
$\mathbf{2 7 0}$ & 5 & $1.11 \times 10^{-3}$ & $1.1 \times 10^{-8}$ & $8.93 \times 10^{-9}$ & $1.2 \times 10^{-10}$ \\
$\mathbf{2 7 0}$ & 6 & $1.33 \times 10^{-3}$ & $9.8 \times 10^{-9}$ & $8.78 \times 10^{-9}$ & $2.2 \times 10^{-10}$ \\
$\mathbf{3 1 6}$ & 2 & $1.85 \times 10^{-3}$ & $9.49 \times 10^{-9}$ & $9.5 \times 10^{-9}$ & $1.8 \times 10^{-10}$ \\
$\mathbf{3 1 6}$ & 3 & $2.78 \times 10^{-3}$ & $7.54 \times 10^{-9}$ & $7.61 \times 10^{-9}$ & $8 \times 10^{-9}$ \\
$\mathbf{3 2 2}$ & 1 & $3.11 \times 10^{-3}$ & $6.38 \times 10^{-9}$ & $5.91 \times 10^{-9}$ & $3.9 \times 10^{-10}$
\end{tabular}

Table 4. Same as Table 2 for the SU of the SUEP instrument

\begin{tabular}{llllll} 
Session & $k$ & $k f_{s t i}$ & $\lambda_{F f}$ & $\lambda_{F t}$ & $\sigma_{t}$ \\
\hline $\mathbf{7 8 2}$ & 1 & $2.22 \times 10^{-4}$ & $2.32 \times 10^{-9}$ & $2.28 \times 10^{-9}$ & $2.1 \times 10^{-12}$ \\
$\mathbf{7 8 2}$ & 2 & $4.44 \times 10^{-4}$ & $2.81 \times 10^{-9}$ & $2.84 \times 10^{-9}$ & $6.2 \times 10^{-12}$ \\
$\mathbf{7 8 2}$ & 3 & $6.66 \times 10^{-4}$ & $2.83 \times 10^{-9}$ & $2.88 \times 10^{-9}$ & $1.3 \times 10^{-11}$ \\
$\mathbf{7 8 2}$ & 4 & $8.88 \times 10^{-4}$ & $2.71 \times 10^{-9}$ & $2.78 \times 10^{-9}$ & $2.4 \times 10^{-11}$ \\
$\mathbf{3 0 6}$ & 1 & $9.24 \times 10^{-4}$ & $2.55 \times 10^{-9}$ & $2.55 \times 10^{-9}$ & $1.2 \times 10^{-11}$ \\
$\mathbf{7 8 2}$ & 5 & $1.11 \times 10^{-3}$ & $2.59 \times 10^{-9}$ & $2.69 \times 10^{-9}$ & $4.4 \times 10^{-11}$ \\
$\mathbf{7 8 2}$ & 6 & $1.33 \times 10^{-3}$ & $2.33 \times 10^{-9}$ & $2.62 \times 10^{-9}$ & $8.7 \times 10^{-11}$ \\
$\mathbf{3 0 6}$ & 2 & $1.85 \times 10^{-3}$ & $2.98 \times 10^{-9}$ & $2.97 \times 10^{-9}$ & $6.2 \times 10^{-11}$ \\
$\mathbf{3 0 6}$ & 3 & $2.78 \times 10^{-3}$ & $2.77 \times 10^{-9}$ & $2.69 \times 10^{-9}$ & $1.5 \times 10^{-9}$ \\
$\mathbf{3 0 0}$ & 1 & $3.11 \times 10^{-3}$ & $5.19 \times 10^{-9}$ & $4.39 \times 10^{-9}$ & $1.9 \times 10^{-10}$
\end{tabular}

Table 5. Same as Table 2 for the SU of the SUREF instrument

The results are presented in Table 2, 5. Each table gathers the results corresponding to the thermal stimulus of the same part (FEEU or SU) on the same instrument (SUEP or SUREF) and are presented by increasing value of the frequency. We can note that, taking into account an uncertainty of $3 \sigma$, the results obtained with the temporal method and with the frequency method are compatible. Moreover there is no obvious dependency of the frequency for the FEEU SUREF and for the SU SUREF. That is not the case for the SU and the FEEU of the SUEP instrument for which the sensitivity clearly decreases when the frequency increases (Figure 10. This can be explained by the fact that the temperature sensor is not placed at the source of temperature leading to thermal inertia. As verified on Figure 10 (solid lines) the frequency dependency can be pretty well modeled by an exponential function.
According to the analysis described in this paper we can adopt conservative values of thermal sensitivities which will be applied, using the measured temperatures during the EP sessions, to compute upper bounds of the systematic effects due to thermal variations. Concerning thermal variations in the FEEU SUREF and in the SU SUREF, our analysis evidence no clear dependence of frequency; that is why we adopt the largest value (and not the mean) given by tables 3 and 5 respectively. For the SUEP we have found a frequency dependency which can be well represented by an exponential function (Figure 10); thus we can apply this function to the frequencies $f_{E P 2}$ and $f_{E P 3}$ which are used in the EP sessions. The values of these sensitivities are reported in Table 6 .

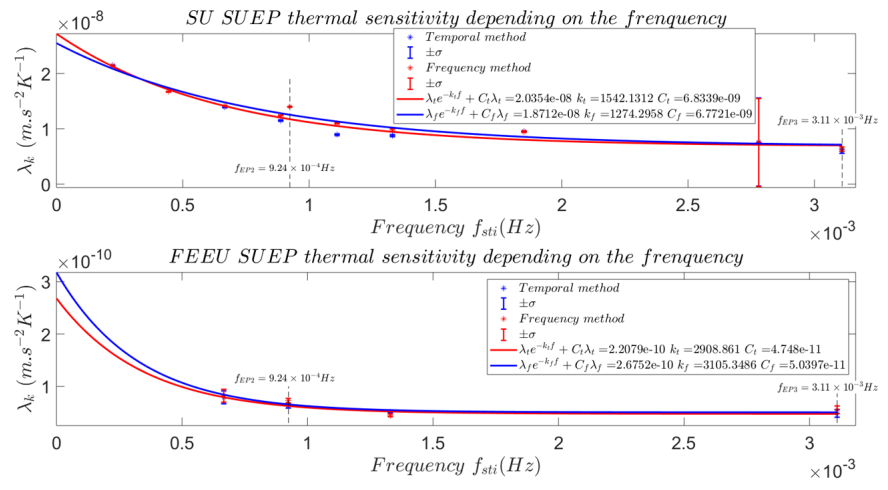

Fig. 10. Sensitivities computed with respect to the frequency for the SU and the FEEU of the SUEP instrument ; representation of the SUEP's SU sensitivity and the SUEP's FEEU sensitivity by an exponential function for both methods

\begin{tabular}{ll} 
& $\lambda\left(m . s^{-2} \cdot K-1\right)$ \\
\hline SU SUEP $\left(f_{E P 2}\right)$ & $1.4 \times 10^{-8}$ \\
SU SUEP $\left(f_{E P 3}\right)$ & $6.4 \times 10^{-9}$ \\
FEEU SUEP $\left(f_{E P 2}\right)$ & $7.3 \times 10^{-11}$ \\
FEEU SUEP $\left(f_{E P 3}\right)$ & $5.5 \times 10^{-11}$ \\
FEEU SUREF & $7.1 \times 10^{-11}$ \\
SU SUREF & $5.2 \times 10^{-9}$
\end{tabular}

Table 6. Final results for thermal sensitivity of each instrument

All these analysis will help us better characterizing the thermal behavior of the instrument. For the final analysis, the values in Table 6 will be used with the temperature fluctuations in order to obtain the maximum contribution of the thermal systematic.

\section{References}

Baghi, Q., Métris, G., Bergé, J., Christophe, B., Touboul, P., \& Rodrigues, M. (2015). Regression analysis with missing data and unknown colored noise: Application to the microscope space mission. Phys. Rev. D, 91, 062003. URL: https://link.aps .org/doi/10.1103/PhysRevD.91. 062003 doi 10.1103/PhysRevD.91.062003

Baghi, Q., Métris, G., Bergé, J., Christophe, B., Touboul, P., \& Rodrigues, M. (2016). Gaussian regression and power spectral density estimation with missing data: The microscope space mission as a case study. Phys. Rev. D, 93, 122007. URL: https://link.aps.org/doi/10.1103/PhysRevD. 93.122007 doi 10.1103/PhysRevD.93.122007 
4 Bergé, J., Baghi, Q., Hardy, E., Métris, G., Robert, A., Rodrigues, M., Touboul, P., Chhun, R., Guidotti, P.-Y., Pires, S., Reynaud, S., Serron, L.,

5 \& Travert, J.-M. (2020). Microscope mission: Data analysis principle. 6 arXiv:2012.06484

7 Chapman, P. K., \& Hanson, A. J. (1970). An Eötvös Experiment in Earth

8 Orbit. R.W. Davies (Ed.), Proc. Conf. on Experimental Tests of Gravitation 9 Theories, JPL TM 33-499, 228.

10 Everitt, C., Damour, T., Nordtvedt, K., \& Reinhard, R. (2003). Historical per11 spective on testing the equivalence principle. Advances in Space Research, 11 32(7), 1297 - 1300. URL: http://www.sciencedirect.com/science/ 12 article/pii/S0273117703903358 doi/https://doi.org/10.1016/ 13 S0273-1177 (03) 90335-8 Fundamental Physics in Space.

14 Mester, J., Torii, R., Worden, P., Lockerbie, N., Vitale, S., \& Everitt, 15 C. W. F. (2001). The STEP mission: principles and baseline de15 sign. Classical and Quantum Gravity, 18(13), 2475-2486. URL: https: 16 //doi.org/10.1088\%2F0264-9381\%2F18\%2F13\%2F310 doi $10.1088 /$ 17 0264-9381/18/13/310

18 Nobili, A., Bramanti, D., Catastini, G., Polacco, E., Milani, A., Anselmo, L., 19 Andrenucci, M., Marcuccio, S., Genovese, A., Genta, G., Brusa, E., \& Prete, 20 C. (1995). Galileo galilei: Flight experiment on the equivalence principle 20 with field emission electric propulsion. J. Astronaut. Sc., 43, 219-242.

21 Nordtvedt, K. (1968). Testing relativity with laser ranging to the moon. Phys.

22 Rev., 170, 1186-1187. URL: https://link.aps.org/doi/10.1103/

23 PhysRev.170.1186 doi 10.1103/PhysRev.170.1186

24 Robert, A., Cipolla, V., Prieur, P., Touboul, P., Métris, G., Rodrigues, M., 25 André, Y., Bergé, J., Boulanger, D., Chhun, R., Christophe, B., Guidotti, 25 P.-Y., Hardy, E., Lebat, V., Lienart, T., Liorzou, F., \& Pouilloux, B.

26 (2020). Microscope satellite and its drag-free and attitude control system.

27 arXiv:2012.06479

28 Touboul, P., Métris, G., Rodrigues, M., André, Y., Baghi, Q., Bergé, J.,

29 Boulanger, D., Bremer, S., Carle, P., Chhun, R., Christophe, B., Cipolla,

30 V., Damour, T., Danto, P., Dittus, H., Fayet, P., Foulon, B., Gageant, C., 31 Guidotti, P.-Y., Hagedorn, D., Hardy, E., Huynh, P.-A., Inchauspe, H., 31 Kayser, P., Lala, S., Lämmerzahl, C., Lebat, V., Leseur, P., Liorzou, F. 32 m. c., List, M., Löffler, F., Panet, I., Pouilloux, B., Prieur, P., Rebray, 33 A., Reynaud, S., Rievers, B., Robert, A., Selig, H., Serron, L., Sum34 ner, T., Tanguy, N., \& Visser, P. (2017). Microscope mission: First re35 sults of a space test of the equivalence principle. Phys. Rev. Lett., 119, 36 231101. URL: https://link.aps.org/doi/10.1103/PhysRevLett. 36119.231101 doi 10.1103/PhysRevLett.119.231101

37 Touboul, P., Métris, G., Rodrigues, M., André, Y., Baghi, Q., Berge, 38 J., Boulanger, D., Bremer, S., Chhun, R., Christophe, B., Cipolla, V., 39 Damour, T., Danto, P., Dittus, H., Fayet, P., Foulon, B., Guidotti, P.-Y., 40 Hardy, E., Huynh, P.-A., Lämmerzahl, C., Lebat, V., Liorzou, F., List, 41 M., Panet, I., Pires, S., Pouilloux, B., Prieur, P., Reynaud, S., Riev1 ers, B., Robert, A., Selig, H., Serron, L., Sumner, T., \& Visser, P. N. 42 (2019). Space test of the Equivalence Principle: first results of the 43 MICROSCOPE mission. Classical and Quantum Gravity, 36, 225006. 44 URL: https://hal.archives-ouvertes.fr/hal-02299493 doi 10. 45 1088/1361-6382/ab4707

46 Touboul, P., Rodrigues, M., Métris, G., Chhun, R., Robert, A., Baghi, Q., 46 Hardy, E., Bergé, J., Boulanger, D., Christophe, B., Cipolla, V., Foulon, B., 47 Guidotti, P.-Y., Huynh, P.-A., Lebat, V., Liorzou, F., Pouilloux, B., Prieur,

48 P., \& Reynaud, S. (2020). Microscope mission analysis, requirements and 49 expected performance. arXiv:2012.06472

50 Touboul, P., Rodrigues, M., Métris, G., \& Tatry, B. (2001). Mi51 croscope, testing the equivalence principle in space. Comptes 52 Rendus de l'Académie des Sciences - Series IV - Physics, 2(9),

521271 - 1286. URL: http://www.sciencedirect.com/science/ 53 article/pii/S1296214701012641 doi https://doi.org/10.1016/ 54 S1296-2147(01)01264-1

55 Viswanathan, V., Fienga, A., Minazzoli, O., Bernus, L., Laskar, J., \& Gastineau, 56 M. (2018). The new lunar ephemeris inpop17a and its application to 56 fundamental physics. Monthly Notices of the Royal Astronomical Soci57 ety, 476(2), 1877-1888. URL: http://dx.doi.org/10.1093/mnras/ 58 sty096 doi $10.1093 / \mathrm{mnras} /$ sty096

59 Wagner, T. A., Schlamminger, S., Gundlach, J. H., \& Adelberger, 60 E. G. (2012). Torsion-balance tests of the weak equivalence principle. Classical and Quantum Gravity, 29(18), 184002. URL: https://doi.org/10.1088\%2F0264-9381\%2F 29\%2F $18 \% 2$ F 184002 